\title{
450 - Exploring staff perspectives on the role of physical environment in dementia care facilities in Sweden and Canada
}

Sook Young Lee; Lillian Hung; Habib Chaudhury

This study explored staff perceptions of the role of physical environment in dementia care facilities in affecting resident's behaviors and staff care practice. We used focus group method (Krueger \& Casey, 2000; Krueger, 1998) to elicit staff's shared perceptions on the impact of the physical environment on residents' behaviors and on their own care practice. A total of 24 staff members from four facilities, two in Sweden and two in Canada, participated. Discussions in the focus groups generated rich and intersubjective accounts via dynamic and interactive exchange among participants. Participants were explained that the researchers were particularly interested in three aspects of the physical environment: architecture or spatial layout of the setting (e.g. corridor length, bath room size, etc.), interior design aspects (e.g. lighting, flooring, furnishing, etc.) and sensory aspects (e.g. noise, smell, tactile properties, etc.). Staff in both countries reported similar physical environmental characteristics that enabled and hindered them from delivering good care. This study yielded three environmental themes that have a substantial effect on the social interaction and care practice: design ambience, space arrangement, and sensory stimuli. The deficits in the physical environmental characteristics prevented staff from providing effective person-centred care. Our findings identified substantial differences between the facilities of the two countries, although it is possible that greater differences might exist between the range of facilities in each country about the quality of environment and care. The quality of environment contributed to a high job satisfaction reported by staff in Sweden. The unsupportive and problematic features of the physical environment seemed to be the primary factor that triggers agitation among the residents with dementia in Canada. 